\title{
Biology meets astronomy
}

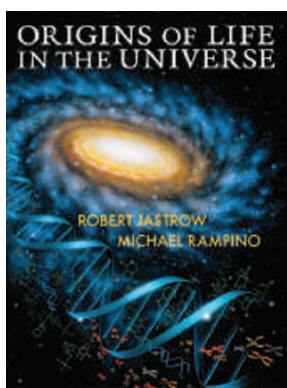

\author{
Origins of Life in \\ the Universe \\ By Robert Jastrow \\ and Michael Rampino
}

CAMBRIDGE UNIV. PRESS: 2009. 395 PP. E24.99; $\$ 50.00$ PAPERBACK

$\wedge$ th strobiology, the study of life in the Solar System and beyond, is a relatively new field that seeks to answer a very old and fundamental question: where did we come from? The subject encompasses such diverse topics as the creation of the basic building blocks of life and the development of an environment that allowed life to flourish here on Earth. It touches on astronomy, geology, geophysics, atmospheric science, chemistry and biology - a challenge for any author.

Yet it is critical that subjects such as astrobiology be presented clearly to non-scientists, particularly because a large section of the public seems to be surprisingly insulated from rapid scientific advances. For example, a poll in 2005 revealed that $51 \%$ of Americans do not believe in evolution. Robert Jastrow (an astronomer) and Michael Rampino (an Earth scientist) have now taken a comprehensive look at astrobiology in an accessible, well-written text aimed at undergraduate students who do not pursue science as a principal subject.

The book starts at the beginning, with the origin of the Universe and the Solar System, to set the foundation for where the building blocks of life came from. In doing so, the authors manage to sneak in a lot of fundamental astronomy, ensuring that their readers will come away with a good basic understanding of how stars and the universe form and evolve. They walk us clearly through cosmological puzzles such as the flatness problem - the enigma about how the early Universe happened to have a density close enough to the critical density to allow galaxies, stars and planets to form. A period of rapid inflation shortly after the Big Bang may provide the answer - all explained in a way that makes the reader feel as though astrophysics isn't that complicated after all. A whirlwind tour of the Solar System is followed by more detailed discussions of the Moon, Mars and Venus, before moving on to a detailed description of our own planet's geological development and the evolution of life on Earth.

Most of the text focuses on Mars as the most likely place in the Solar System to look for life beyond Earth, but at the end, the authors expand into the search for intelligent extraterrestrial life outside (and whether it actually exists). Given our own inability to go much beyond Earth, except with our radio and TV signals, our seeming aloneness should not be a puzzle - but try telling that to those who interpret crop circles as evidence for aliens!

Its completeness is the book's strength. No aspect relating to the origins of life in the Universe is left uncovered, and the authors are particularly thorough in their discussion of the origin and evolution of our habitable and inhabited Earth. The authors walk the student through the basics of genetics, how life may have originated, and then through mechanisms as well as processes of evolution.

The book's weak point is its organization. A lot of emphasis is placed on the origin and evolution of the Universe and the Solar System; however, the equally or more exciting possibility of life existing in the outer Solar System, for example, on Jupiter's moon Europa, is only mentioned in passing. The implications of life in extreme terrestrial environments for life elsewhere are also discussed only briefly. Furthermore, the order of the text does not always help to explain the logic behind our current search for life beyond Earth.

Of course some minor errors are hard to avoid in such a comprehensive book. For example, the contributions of Pioneer Venus - an orbiter that was launched in the late 1970s - to our understanding of the atmosphere of Venus are incorrectly attributed to the Magellan spacecraft. The contributions of the Soviet Union to our knowledge of Venus, in particular through surface exploration by the Venera landers, are neglected altogether. In addition, more recent suggestions that water may have persisted on the surface of Venus for several thousand million years - raising the possibility of the development of life on early Venus - are omitted.

Despite these issues, this book gives an extremely comprehensive understanding of the evolution of life and the possibility for life beyond our own planet, and it should serve as an excellent textbook for an introductory astrobiology course. As each day passes and fresh data come back on newly discovered extra-solar planets, geysers on Enceladus, lakes on Titan with unique compositions and fresh evidence for a watery past on Mars, the hard part is keeping track of the latest, greatest place to search for life beyond Earth.

\section{REVIEWED BY ELLEN STOFAN \\ Ellen Stofan is a Senior Research Scientist with Proxemy Research, PO Box 338, Rectortown, \\ Virginia 20140, USA \\ e-mail:estofan@rcn.com}

\section{Heavenly rinds}

C omprising a planet's outermost portion, the crust is by far its most accessible part, and in the case of Earth it is of critical importance to sustaining life. However, the bewildering array of worlds in the Solar System means that in spite of this accessibility, a coherent understanding of crusts of the solid planets has not yet emerged. In their treatise, S. Ross Taylor and Scott McLennan have attempted to systematically survey the available evidence and current theory for crustal formation in all the observed bodies in the Solar System, and to construct a framework for understanding their diversity.

This work can be thought of as an extension of their previous book, The Continental Crust: Its Composition and Evolution (1985), taking advantage of several decades of planetary and terrestrial research to broaden its scope. The emphasis is on the Moon, Mars and Earth, with considerable attention to Mercury and Venus; the numerous satellites of the outer planets and the minor planets such as asteroids (particularly 4 Vesta) and trans-Neptunian objects (for example Pluto/Charon and Eris) receive cursory treatment. 